\title{
On Numerical Solution of the Incompressible Navier-Stokes Equations with Static or Total Pressure Specified on Boundaries
}

\author{
N. P. Moshkin and D. Yambangwai \\ School of Mathematics, Suranaree University of Technology, Nakhon Ratchasima 30000, Thailand \\ Correspondence should be addressed to N. P. Moshkin, nikolay.moshkin@gmail.com
}

Received 14 October 2008; Revised 9 February 2009; Accepted 27 March 2009

Recommended by Saad A. Ragab

\begin{abstract}
The purpose of this article is to develop and validate a computational method for the solution of viscous incompressible flow in a domain with specified static or total pressure on the flow-through boundaries (inflow and outflow). The computational algorithm is based on the Finite Volume Method in nonstaggered boundary-fitted grid. The implementations of the boundary conditions on the flow-through parts of the boundary are discussed. Test examples illustrate the main features and validity of the proposed method to study viscous incompressible flow through a bounded domain with specified static pressure (or total pressure) on boundary as a part of well-posed boundary conditions.
\end{abstract}

Copyright (C) 2009 N. P. Moshkin and D. Yambangwai. This is an open access article distributed under the Creative Commons Attribution License, which permits unrestricted use, distribution, and reproduction in any medium, provided the original work is properly cited.

\section{Introduction}

A flow of a viscous incompressible fluid through a given domain is rather interesting for its numerous engineering applications. Typically, these include tube and channel flows with a variety of geometries. The difficulties in mathematical modeling and numerical simulation of such flows arise in the flow-through boundaries (inflow and outflow). If the domain of interest is completely bounded by impermeable walls, there is no ambiguity in the boundary conditions for the incompressible Navier-Stokes equations. However, when flow-through (inflow and outflow) boundaries are present, there is no general agreement on which kind of boundary conditions is both mathematically correct and physically appropriate on these flow-through boundaries. Traditionally, such problems are treated with specified velocity on the domain boundaries. However, in many applications the boundary velocities are not known; instead the pressure variation is given at the boundaries, and the flow within the domain has to be determined. For example, in the central air-conditioning or air-heating system of a building, a main supply channel branches into many subchannels that finally open into the different rooms, which can be at a different constant pressure. The distribution 
of the flow into various branches depends on the flow resistances of these branches, and in general, it is even impossible to predict the direction of flow.

The problem of solvability and uniqueness of an initial boundary value problem for the incompressible Navier-Stokes equations is one of the various problems considered, for example, in [1-6] and many others. Major part of research deals with proper formulation of boundary conditions for pressure which are needed in numerical simulation but absence in the mathematical statement of problem (see, e.g., more recent $[7,8]$ and therein references). The object of our study is a boundary value problem in which the pressure is known on boundary as a part of boundary conditions in the mathematical statement of problem.

Antontsev et al. [1], Ragulin [4], and Ragulin and Smagulov [5] have studied initial boundary value problems in which the values of pressure or total pressure are specified on flow-through boundaries. Ragulin [4] and Ragulin and Smagulov [5] have considered problems for the homogeneous Navier-Stokes equations. Antontsev et al. [1] have studied well-posedness of the nonhomogeneous Navier-Stokes equations. As these results are not well known, we will shortly represent the well-posed statement of initial boundary value problems with specified pressure boundaries.

To the best of the authors' knowledge, the research on numerically treated pressure boundary conditions for the incompressible Navier-Stokes equations is limited. Some of the research conducted is discussed below. Kuznetsov et al. [9] and Moshkin [10-12] developed finite difference algorithms to treat incompressible viscous flow in a domain with given pressure on flow-through parts of the boundary. Finite-difference numerical algorithms were developed for primitive variables and for stream function vorticity formulation of $2 D$ NavierStokes equations.

In the finite-element study by Hayes et al. [13], a brief discussion of the specified pressure on the outflow region of the boundary is presented. Kobayashi et al. [14] have discussed the role of pressure specified on open boundaries in the context of the SIMPLE algorithm.

The prescription of a pressure drop between the inlet and the outlet of the flow was also considered by Heywood et al. [15], where a variational approach with given mean values of the pressure across the inflow and outflow boundaries was used.

The construction of the discretized equations for unknown velocities on specified pressure boundaries and the solution of the discretized equations using the SIMPLE algorithm are discussed in [16]. The computational treatment of specified pressure boundaries in complex geometries is presented within the framework of a nonstaggered technique based on curvilinear boundary-fitted grids. The proposed method is applied for predicting incompressible forced flows in branched ducts and in buoyancy-driven flows.

A finite-difference method for solving the incompressible time-dependent threedimensional Navier-Stokes equations in open flows where Dirichlet boundary conditions for the pressure are given on part of the boundary is presented in [17]. The equations in primitive variables (velocity and pressure) are solved using a projection method on a nonstaggered grid with second-order accuracy in space and time. On the inflow and outflow boundaries the pressure is obtained from its given value at the contour of these surfaces using a twodimensional form of the pressure Poisson equation, which enforces the incompressibility constraint $\nabla \cdot v=0$. The pressure obtained on these surfaces is used as Dirichlet boundary conditions for the three-dimensional Poisson equation inside the domain. The solenoidal requirement imposes some restrictions on the choice of the open surfaces.

Barth and Carey [18] discussed the choice of appropriate inflow and outflow boundary conditions for Newtonian and generalized Newtonian channel flows. They came 


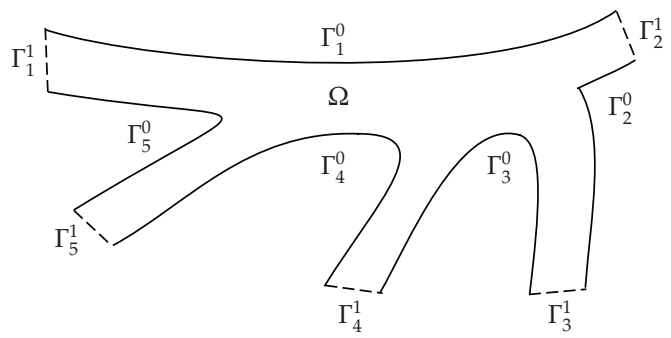

Figure 1: Sketch of the flowing-through domain.

to conclusion that "...For real-world problems that are fundamentally pressure driven and involve complex geometries, it is desirable to impose a pressure drop by means of specified pressures at the inflow and outflow boundaries..." At the inflow and outflow boundaries one of the conditions specifies the normal component of the surface traction force, and the other two imply that there is no tangential flow at these boundaries; that is, flow is normal to the inflow and outflow boundaries. But no mathematical justification was given.

Let us call problems where fluid can enter or leave a domain through parts of the boundary, a "flowing-through problem" for viscous incompressible fluid flow. In [17] these problems are called problems with "open" boundaries. We think that the term flowingthrough problem is more suitable. The purpose of our research is not to add new insight into the mathematical statement of the problem but to develop a finite volume method for solving a flowing-through problem for the incompressible Navier-Stokes equations for which questions of existence and uniqueness have been considered in $[1,4,5]$.

In the following sections of this paper, a brief overview of various kinds of well-posed flowing-through problems for the incompressible Navier-Stokes equations is presented. This is followed by a description of the finite volume numerical method with strength on implementation of boundary conditions on the flow-through parts. The numerical method is then validated by a comparison of analytical and numerical solutions for the laminar flow driven by pressure drop in the $2 D$ plane channel, in the $2 D$ gap between two cylinders, in U-bend channel, and in a planar T-junction channel.

\section{Mathematical Formulation of Flowing-Through Problems}

We present here the various kinds of well-posed flowing-through boundary value problems for the incompressible Navier-Stokes equation. In our explanation, we follow Antontsev et al. [1], Ragulin [4], and Ragulin and Smagulov [5]. Let us consider the flow of viscous liquid through bounded domain $\Omega$ of $R^{d}(d=2$ or 3$), t \in[0, T]$, where $T>0$ is a fixed time. Let $\Gamma_{k^{\prime}}^{1} \quad k=1, \ldots, K$ denote parts of the boundary $\Gamma=\partial \Omega$ where the fluid enter or leave the domain. Let $\Gamma_{l}^{0}, l=1, \ldots, L$ be an impermeable parts of the boundary, $D=\Omega \times(0, T)$, $S=\Gamma \times(0, T), S_{i}^{\alpha}=\Gamma_{i}^{\alpha} \times(0, T), \alpha=0,1$. Scheme of the domain is depicted in Figure 1.

The flowing-through problem is to find a solution of the Navier-Stokes equations

$$
\begin{aligned}
\frac{\partial \vec{u}}{\partial t}+(\vec{u} \cdot \nabla) \vec{u} & =-\frac{1}{\rho} \nabla p+v \Delta \vec{u}, \\
\nabla \cdot \vec{u} & =0,
\end{aligned}
$$


in the domain $D=\Omega \times(0, T)$ with appropriate initial and boundary conditions, where $\vec{u}$ is the velocity vector, $p$ is the pressure, $\rho$ is the density, and $v$ is the kinematic viscosity. The initial data are

$$
\left.\vec{u}\right|_{t=0}=\vec{u}^{0}(\vec{x}), \quad \nabla \cdot \vec{u}^{0}=0, \quad \vec{x} \in \Omega
$$

On the solid walls $\Gamma_{l}^{0}$, the no-slip condition holds

$$
\vec{u}=0, \quad(\vec{x}, t) \in S_{l}^{0}, l=1, \ldots, L
$$

On the flow-through parts $\Gamma_{k}^{1}, k=1, \ldots, K$ three types of boundary conditions can be set up to make problem wellposed. As shown in $[1,4]$, the conditions are the followings.

(i) On the flow-through parts $\Gamma_{j}^{1}, j=j_{1}, \ldots, j_{n}$, the tangent components of the velocity vector and the total pressure are prescribed as

$$
\begin{gathered}
\vec{u} \cdot \vec{\tau}_{m}=G_{j}^{m}(\vec{x}, t), \quad m=1,2, \\
p+\frac{1}{2} \rho|\vec{u} \cdot \vec{u}|=H_{j}(\vec{x}, t), \quad(\vec{x}, t) \in S_{j}^{1}, j=j_{1}, \ldots, j_{n} .
\end{gathered}
$$

Here $\vec{\tau}_{1}, \vec{\tau}_{2}$ are the linearly independent vectors tangent to $\Gamma_{j}^{1}$. Functions $G_{j}^{m}(\vec{x}, t)$, and $H_{j}(\vec{x}, t)$ are given on $S_{j}^{1}=\Gamma_{j}^{1} \times(0, T)$.

(ii) On the flow-through parts $\Gamma_{l}^{1}, l=l_{1}, \ldots, l_{n}$, the tangent components of the velocity vector and pressure are known as

$$
\vec{u} \cdot \vec{\tau}_{m}=G_{l}^{m}(\vec{x}, t), \quad m=1,2, \quad p=H_{l}(\vec{x}, t), \quad(\vec{x}, t) \in S_{l}^{1}, l=l_{1}, \ldots, l_{n} .
$$

Here $G_{l}^{m}(\vec{x}, t)$ and $H_{l}(\vec{x}, t)$ are given on $S_{l}^{1}=\Gamma_{l}^{1} \times(0, T)$.

(iii) On the flow-through parts $\Gamma_{s}^{1}, s=s_{1}, \ldots, s_{n}$, the velocity vector (all three components) has to be prescribed as

$$
\vec{u}=\vec{u}_{s}^{1}(\vec{x}, t), \quad(\vec{x}, t) \in S_{s}^{1}, \quad s=s_{1}, \ldots, s_{n}
$$

Here $\vec{u}_{s}^{1}(\vec{x}, t)$ is given on $S_{s}^{1}=\Gamma_{s}^{1} \times(0, T)$.

It should be mentioned that various combinations of boundary conditions on $S_{k^{\prime}}^{1} k=$ $1, \ldots, K$ give well-posed problems. For example, on the portion of the flow-through parts $S_{j}^{1}, j=j_{1}, \ldots, j_{n}$ one kind of boundary condition may hold, and other portions another kinds may hold.

\section{Finite Volume Approximation of Flowing-Through Problems}

Let us present the numerical algorithm for the flowing-through problem. Numerous variation of projection methods have been developed and have been successfully utilized in computing 
incompressible flow problems. To emphasize on pressure boundary conditions, we used here simple explicit projection method. Although some of the main aspects are well known in literature, for the sake of completeness details are given.

\subsection{Time Discretization}

The time discretization used here is based upon the simplest projection scheme originally proposed by Chorin and Temam (see, e.g., $[19,20]$ ). This scheme has an irreducible splitting error of order $O(\Delta t)$. Hence, using a higher-order time stepping scheme for the operator $\partial / \partial t-v \Delta$ does not improve overall accuracy. Using the explicit Euler time stepping, the marching steps in time are the following.

Set $\left.\vec{u}\right|_{t=0}=\vec{u}^{0}$, then for $n \geq 0$ compute $\vec{u}^{*}, \vec{u}^{n+1}$, and $p^{n+1}$ by solving first substep:

$$
\frac{\vec{u}^{*}-\vec{u}^{n}}{\Delta t}+\left(\vec{u}^{n} \cdot \nabla\right) \vec{u}^{n}=v \Delta \vec{u}^{n}
$$

and second substep:

$$
\begin{array}{r}
\frac{\vec{u}^{n+1}-\vec{u}^{*}}{\Delta t}=-\nabla p^{n+1}, \\
\nabla \cdot \vec{u}^{n+1}=0, \quad\left(\vec{u}^{n+1}\right)_{\Gamma^{0}}=0,
\end{array}
$$

where $\Delta t=T / N$ is the time step, $N$ is the integer, $\vec{u}^{n} \approx \vec{u}(\vec{x}, n \Delta t)$, and $p^{n+1} \approx p(\vec{x},(n+$ 1) $\Delta t$ ). Without loss of generality, density is equal to one, $\rho=1$. The explicit approximation of convective and viscous terms in (3.1) introduces restriction on the time step for stability. This is analyzed by many (see, e.g., [20,21] and therein references).

\subsection{Space Discretization}

For the sake of simplicity and without loosing generality, the formulation of the numerical algorithm is illustrated for a two-dimensional domain. Let $\vec{u}=\left(u_{x}, u_{y}\right)$ be the velocity vector, where $u_{x}$ and $u_{y}$ are the Cartesian components in $x$ and $y$ direction, respectively. The finite volume discretization is represented for nonorthogonal quadrilaterals grid. The collocated variable arrangement is utilized. Each discrete unknown is associated with the center of control volume $\Omega$. First, we discretize the convection and diffusion parts of the Navier-Stokes equation. One can recast (3.1) in the form

$$
\frac{\phi^{*}-\phi^{n}}{\Delta t}+\nabla \cdot\left(\phi^{n} \vec{u}^{n}\right)=v \Delta \phi^{n}
$$

where the variable $\phi$ can be either $u_{x}$ or $u_{y}$, and $\vec{u}^{n}$ is such that $\nabla \cdot \vec{u}^{n}=0$. 


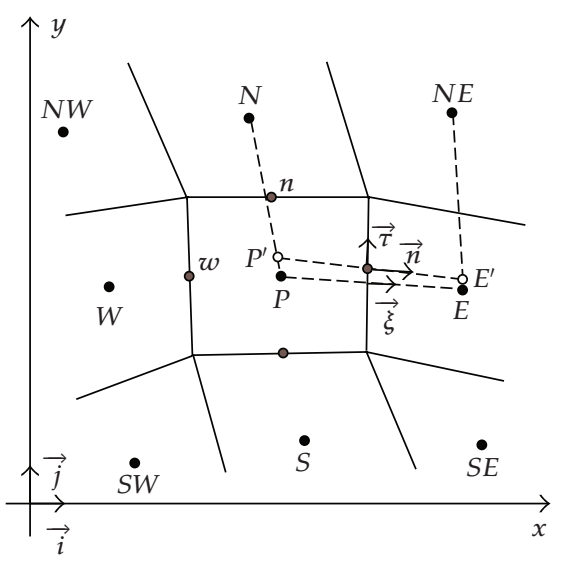

(a)

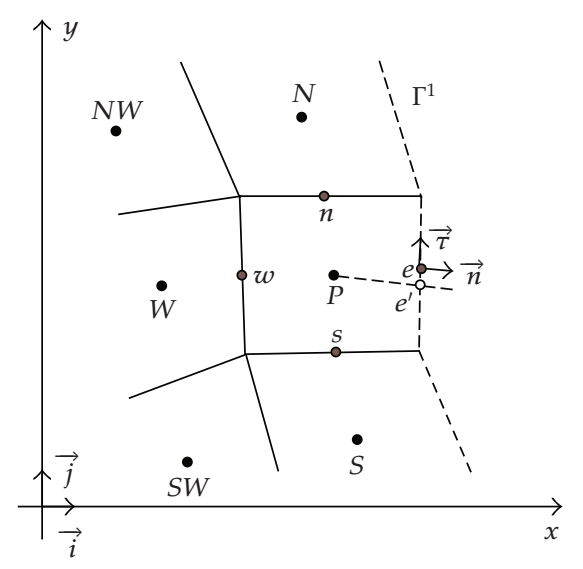

(b)

Figure 2: A typical 2D control volume and the notation used. A way of calculating cell face values and gradients.

The discrete form of (3.4) is obtained by integrating on each control volume $\Omega$, followed by the application of the Gauss theorem:

$$
\int_{\Omega} \frac{\phi^{*}-\phi^{n}}{\Delta t} d \Omega+\oint_{S} \phi^{n}\left(\vec{u}^{n} \cdot \vec{n}\right) d S=v \oint_{S} \nabla \phi^{n} \cdot \vec{n} d S
$$

where $S$ is the boundary of control volume $\Omega$ (e.g., in the case shown in Figure 2, $S$ is the union of the control volume faces $s, e, n$, and $w$ ), and $\vec{n}$ is the unit outward normal vector to $S$. Using the midpoint rule to approximation, the surface and volume integrals yield

$$
\begin{gathered}
\int_{\Omega} \frac{\phi^{*}-\phi^{n}}{\Delta t} d \Omega \approx\left(\frac{\phi^{*}-\phi^{n}}{\Delta t}\right)_{P} \Delta \Omega, \\
\oint_{S} \phi^{n}\left(\vec{u}^{n} \cdot \vec{n}\right) d S \approx \sum_{c=e, s, n, w} \phi_{c}^{n}\left(\vec{u}^{n} \cdot \vec{n}\right)_{c} S_{c}, \\
\oint_{S} \nabla \phi^{n} \cdot \vec{n} d S=\oint_{S} D_{n} \phi^{n} d S \approx \sum_{c=e, s, n, w}\left(D_{n} \phi^{n}\right)_{c} S_{c},
\end{gathered}
$$

where $\Delta \Omega$ is the volume of control volume $\Omega, S_{c}$ is the area of the " $c$ " control volume face, and $\left(D_{n} \phi\right)_{c}$ is the derivative of Cartesian velocity components in the normal direction at the center of the " $c$ " control volume face. To estimate the right-hand side in (3.7) and (3.8), we need to know the value of Cartesian velocity components and its normal derivative on the faces of each control volume. The implementation of Cartesian velocity components on nonorthogonal grids requires special attention because the boundary of the control volume is usually not aligned with the Cartesian velocity components. The $2 D$ interpolation of irregularly-spaced data (see, e.g., [22]) is used to interpolate Cartesian velocity components on the boundary of each control volume in (3.7). Only the east side of a $2 D$ control volume shown in Figure 2(a) will be considered. The same approach applies to other faces, only the 
indices need to be changed. For example, let $\phi_{k}$ be the value of Cartesian velocity components at point $k$ where $k=N, P, S, S E, E, N E$, and let $L_{(e, k)}$ be the Cartesian distance between $e$ and $k$. Using $2 D$ interpolation yields

$$
\phi_{e}=\frac{\left(\sum_{k} L_{(e, k)}^{-2} \phi_{k}\right)}{\left(\sum_{k} L_{(e, k)}^{-2}\right)}, \quad k=N, P, S, S E, E, N E,
$$

where $L_{(e, k)}^{-2}=1 /\left[\left(x_{e}-x_{k}\right)^{2}+\left(y_{e}-y_{k}\right)^{2}\right]$. The derivative of Cartesian velocity components in the normal direction at the center of the control volume face in (3.8) can be calculated by using the central difference approximation (see Figure 2(a)):

$$
\left(D_{n} \phi\right)_{e} \approx \frac{\phi_{E^{\prime}}-\phi_{P^{\prime}}}{L_{\left(P^{\prime}, E^{\prime}\right)}}
$$

The auxiliary nodes $P^{\prime}$ and $E^{\prime}$ lie at the intersection of the line passing through the point " $e$ " in the direction of normal vector $\vec{n}$ and the straight lines which connect nodes $P$ and $N$ or $E$ and $N E$, respectively, and $L_{\left(P^{\prime}, E^{\prime}\right)}$ stands for the distance between $P^{\prime}$ and $E^{\prime}$. The values of $\phi_{E^{\prime}}$ and $\phi_{P^{\prime}}$ can be calculated by using the gradient at control volume center:

$$
\phi_{E^{\prime}}=\phi_{E}+\nabla \phi_{E} \cdot\left(\vec{x}_{E^{\prime}}-\vec{x}_{E}\right), \quad \phi_{P^{\prime}}=\phi_{P}+\nabla \phi_{P} \cdot\left(\vec{x}_{P^{\prime}}-\vec{x}_{P}\right),
$$

where $\vec{x}_{P}, \vec{x}_{E}, \vec{x}_{P^{\prime}}$, and $\vec{x}_{E^{\prime}}$ are the radius vectors of $P, E, P^{\prime}$, and $E^{\prime}$, respectively. The $k$ th Cartesian components of $\nabla \phi_{P}$ are approximated using Gauss's theorem:

$$
\nabla \phi_{P} \cdot \vec{i}_{k}=\left(\frac{\partial \phi}{\partial x_{k}}\right)_{P}=\frac{1}{\Delta \Omega} \sum_{c=e, s, n, w} \phi_{c}^{n+1} S_{c}^{k}, \quad S_{c}^{k}=S_{c}\left(\vec{n} \cdot \overrightarrow{i_{k}}\right)
$$

where $S_{c}$ is the area of " $c$ " control volume face, $\vec{n}$ is the unit outward normal vector to $S_{c}$, and $\vec{i}_{k}$ is the unit basis vector of Cartesian coordinate system $\left(x_{1}, x_{2}\right)=(x, y)$. Using (3.6)-(3.12) to approximate (3.5), one can determine velocity field $\vec{u}^{*}$ (which is not solenoidal) at each grid node, even on the boundary.

In the first substep the continuity (3.3) is not used so that the intermediate velocity field is, in general, nondivergence free. The details of the setting and discretization of the second substep developed on nonuniform, collocated grid are discussed below. Equation (3.2) applies both in continuous and discrete sense. Taking the divergence of both sides of (3.2) and integrating over a control volume $\Omega$, after applying the Gauss theorem and setting the update velocity filed, $\vec{u}^{n+1}$, to be divergence free, one gets the equation

$$
0=\frac{1}{\Delta \Omega} \oint_{S} \vec{u}^{n+1} \cdot \vec{n} d S=\frac{1}{\Delta \Omega} \oint_{S} \vec{u}^{*} \cdot \vec{n} d S-\Delta t \frac{1}{\Delta \Omega} \oint_{S} \nabla p^{n+1} \cdot \vec{n} d S
$$

that has to be discretized while collocating the variables in the control volume centers. Here $\vec{n}$ is outward normal to the boundary $S$ of control volume $\Omega$. At this stage of the projection procedure, the discrete values of $u_{x}^{*}$ and $u_{y}^{*}$ are already known and represent the source term 
in (3.13). A second-order discretization of the surface integrals can be obtained by utilizing the mean value formula. This means that the surface integrals in (3.13) can be approximated as

$$
\begin{aligned}
& \frac{1}{\Delta \Omega} \oint_{S} \vec{u}^{n+1} \cdot \vec{n} d S \cong \frac{1}{\Delta \Omega} \sum_{c=e, s, w, n}\left(\vec{u}^{n+1} \cdot \vec{n}\right)_{c} S_{c} \\
& \frac{1}{\Delta \Omega} \oint_{S} \nabla p^{n+1} \cdot \vec{n} d S \cong \frac{1}{\Delta \Omega} \sum_{c=e, s, w, n}\left(\nabla p^{n+1} \cdot \vec{n}\right)_{c} S_{c} .
\end{aligned}
$$

It follows that by substituting (3.14) into (3.13), one gets the discrete pressure equation

$$
\frac{1}{\Delta \Omega} \sum_{c=e, s, w, n}\left(\vec{u}^{*} \cdot \vec{n}\right)_{c} S_{c}-\frac{\Delta t}{\Delta \Omega} \sum_{c=e, s, w, n}\left(D_{n} p^{n+1}\right)_{c} S_{c}=0
$$

The iterative method is utilized to approximate $\left(D_{n} p^{n+1}\right)_{c}$ and solve (3.15). The normal-toface intermediate velocities $\left(\vec{u}^{*} \cdot \vec{n}\right)_{c}, c=e, s, w, n$ are not directly available. They are found using interpolation. The derivative of pressure with respect to the direction of the outward normal $\vec{n}$ through the cell face " $c$ ", $\left(D_{n} p\right)_{c}^{n+1}$ is approximated by on iterative technique (see, e.g., [23]) to reach a higher order of approximation and preserved compact stencil in the discrete equation (3.15). Only the east face of a $2 D$ control volume shown in Figure 2(a) will be considered. The same approach applies to other faces. Using second upper index " $s$ " to denote the number of iteration, one writes

$$
\begin{gathered}
\left(D_{n} p\right)_{e}^{n+1, s+1}=\left(D_{\xi} p\right)_{e}^{n+1, s+1}+\left[\left(D_{n} p\right)_{e}-\left(D_{\xi} p\right)_{e}\right]^{n+1, s}, \quad s=0, \ldots, \widehat{S}, \\
\left(D_{n} p\right)^{n+1,0}=\left(D_{n} p\right)^{n},
\end{gathered}
$$

where $\xi$ is the direction along the line connecting nodes $P$ and $E$ (see Figure 2(a)). The terms in the square brackets are approximated with high order and are evaluated by using values known from the previous iteration. Once the iterations converge, the low-order approximation term $\left(D_{\xi} p\right)_{e}^{n+1, s+1}$ drops out, and the solution obtained corresponds to the higher order of approximation. The derivatives of pressure in the square brackets are written as

$$
\left(D_{n} p\right)_{e}^{n+1, s}=(\nabla p \cdot \vec{n})_{e}^{n+1, s}, \quad\left(D_{\xi} p\right)_{e}^{n+1, s}=(\nabla p \cdot \vec{\xi})_{e}^{n+1, s}
$$

where $\vec{n}$ is the unit outward normal vector to cell face " $e$ ", and $\vec{\xi}$ is the unit vector in $\xi$ direction from point $P$ to $E$. The term $(\nabla p)_{e}^{n+1, s}$ is approximated similar to (3.9) as

$$
(\nabla p)_{e}^{n+1, s}=\frac{\left(\sum_{l} L_{(e, l)}^{-2} \nabla p_{l}^{n+1, s}\right)}{\left(\sum_{l} L_{(e, l)}^{-2}\right)}, \quad l=N, P, S, S E, E, N E
$$


where $\nabla p_{l}^{n+1, s}$ is the gradient of the pressure at grid node $l$ and $L_{(e, l)}^{-2}=1 /\left[\left(x_{e}-x_{l}\right)^{2}+\left(y_{e}-y_{l}\right)^{2}\right]$. The $k$ th components of $\nabla p_{l}^{n+1, s}$ are discretized by using Gauss theorem (e.g., at grid node $P$ ):

$$
\nabla p_{P}^{n+1, s} \cdot \vec{i}_{k}=\left(\frac{\partial p^{n+1, s}}{\partial x_{k}}\right)_{P} \cong \frac{1}{\Delta \Omega} \sum_{c=e, s, n, w} p_{c}^{n+1, s} S_{c}^{k}, \quad S_{c}^{k}=S_{c}\left(\vec{n} \cdot \overrightarrow{i_{k}}\right) .
$$

The first term in the right-hand side of (3.16) is treated implicitly, and a simple approximation is used (that gives a compact stencil):

$$
\left(D_{\xi} p\right)_{e}^{n+1, s+1} \approx \frac{p_{E}^{n+1, s+1}-p_{P}^{n+1, s+1}}{L_{(P, E)}}
$$

where $L_{(P, E)}$ is the distance between nodes $P$ and $E$. The final expression for the approximation of the derivative of pressure with respect to $\vec{n}$ through the cell face " $e$ " can now be written as

$$
\left(D_{n} p\right)_{e}^{n+1, s+1}=\frac{p_{E}^{n+1, s+1}-p_{P}^{n+1, s+1}}{L_{(P, E)}}+\nabla p^{n+1, s} \cdot(\vec{n}-\vec{\xi})_{e}
$$

The terms labeled " $n+1$, s" become zero when $\vec{\xi}=\vec{n}$ is required. Repeating steps similar to (3.16)-(3.21) for other faces of control volume and substitute result into (3.15), one generates the equation for finding the pressure at next iteration $(n+1, s+1)$ as

$$
\begin{aligned}
& \frac{1}{\Delta \Omega} \sum_{c=e, s, w, n}\left(\vec{u}^{*} \cdot \vec{n}\right)_{c} S_{c}-\frac{\Delta t}{\Delta \Omega} \sum_{c=e, s, w, n}\left(\nabla p^{n+1, s}\right)_{c}(\vec{n}-\vec{\xi})_{c} \\
& \quad=\frac{\Delta t}{\Delta \Omega}\left\{\left(\frac{p_{E}-p_{P}}{L_{(P, E)}}\right)^{n+1, s+1}-\left(\frac{p_{P}-p_{W}}{L_{(P, W)}}\right)^{n+1, s+1}+\left(\frac{p_{N}^{n+1, s}-p_{P}^{n+1, s+1}}{L_{(P, N)}}\right)-\left(\frac{p_{P}-p_{S}}{L_{(P, S)}}\right)^{n+1, s+1}\right\} .
\end{aligned}
$$

We use $p_{N}^{n+1, s}$ instead of $p_{N}^{n+1, s+1}$ to make matrix of algebraic system to be tridiagonal.

\subsection{Implementation of Boundary Conditions}

The Finite Volume Method requires the boundary fluxes for each control volume to be either known or expressed through known quantities and interior nodal values. If the variables values are known at some boundary point, then there is no need to solve problem for it. A difficulty arises when approximations of normal derivatives are needed. Usually (see, e.g., [23]) these derivatives are approximated with lower order than the approximations used for interior point and may be one-sided differences. The accuracy of the results depended not only on the approximation near boundary but also on the accuracy of approximations at interior points. If higher accuracy is required, one has to use higher-order one-sided finite differences of derivatives at boundary and higher-order approximations at interior point. We used first-order one-sided finite differences near boundary. 


\section{Impermeable Wall}

The following condition is prescribed on the impermeable wall:

$$
\vec{u}=\vec{u}_{\text {wall }}
$$

This condition follows from the fact that a viscous fluid sticks to a solid wall. Since there is no flow through the wall, mass fluxes and convective fluxes of all quantities are zero. Diffusive fluxes in the momentum equation are approximated using known boundary values of the unknown and one-sided finite difference approximation for the gradients.

\section{Flow-Through Part}

The implementations of three kinds of boundary conditions on the flow-through parts are addressed here. Only the case where the east face of the control volume aligns with flowthrough boundary $\Gamma^{1}$ will be considered. A sketch of the grid and the notations used are shown in Figure 2(b). Other faces are treated similar.

(a) The velocity is set up (see (2.6)) as

$$
\vec{u}_{\Gamma^{1}}=\vec{u}_{s}^{1}(\vec{x}, t)
$$

Since the velocity vector is given, the mass flow rate and the convective fluxes can be calculated directly. The diffusive fluxes are not known, but they are approximated using known boundary values of the unknowns and one-sided finite difference approximation for the gradient. It is important to note how boundary condition (3.24) is involved in the derivation of the discrete pressure equation. Because $\left(\vec{u}^{n+1}\right)_{e}$ is given by (3.24), the approximation of (3.13) becomes

$$
\frac{1}{\Delta \Omega}\left[\left(\vec{u}^{n+1} \cdot \vec{n}\right)_{e}+\sum_{c=s, w, n}\left(\vec{u}^{*} \cdot \vec{n}\right)_{c} S_{c}\right]-\frac{\Delta t}{\Delta \Omega} \sum_{c=s, w, n}\left(D_{n} p^{n+1}\right)_{c} S_{c}=0 .
$$

One does not need to approximate $\left(D_{n} p^{n+1}\right)_{e}$ at face " $e$ ". However, if pressure at the boundary $\Gamma^{1}$ is needed at some stage, it can be obtained by extrapolation within the domain.

(b) The tangential velocity and pressure are prescribed (see, (2.5)) as

$$
(\vec{u} \cdot \vec{\tau})_{\Gamma^{1}}=G(x, y, t), \quad p_{\Gamma^{1}}=H(x, y, t)
$$

When the tangential velocity and pressure are specified on the flow-through part of boundary, the mass and convective fluxes are not known. One has to find them during the solution process. The solenoidal constraint $\nabla \cdot \vec{u}=0$ has to be applied at the boundary where the pressure is specified. Because the flow-through boundaries may not be aligned with the Cartesian coordinates, we will refer to the local coordinate system $(n, \tau)$ which is a rotated Cartesian frame with $n$ in the 
direction of normal vector to the flow-through boundary and $\tau$ in the direction of the tangential vector to the flow-through boundary. The velocity vector $\vec{u}=\left(u_{x}, u_{y}\right)$ can be expressed in terms of velocity components in local orthogonal coordinates $\vec{u}=\left(U_{n}, U_{\tau}\right)$, where $U_{n}=\vec{u} \cdot \vec{n}$ is the normal velocity component to the flowthrough boundary, and $U_{\tau}=\vec{u} \cdot \vec{\tau}$ is the tangential velocity component to the flow-through boundary which is known at $\Gamma_{1}$ from boundary condition (3.26). The continuity equation in terms of local orthogonal coordinates $(n, \tau)$ reads

$$
\frac{\partial U_{n}}{\partial n}+\frac{\partial U_{\tau}}{\partial \tau}=0
$$

Using (3.26) and (3.27) yields

$$
\left(\frac{\partial U_{n}}{\partial n}\right)_{\Gamma^{1}}=-\frac{\partial G}{\partial \tau}
$$

To find the flux on the flow-through part, one needs to calculate the normal velocity $\left(U_{n}\right)_{e}$ at the east cell face " $e$ " (See Figure 2(b)). The normal derivative of $U_{n}$ at the east cell face is approximated by one-side difference:

$$
\left(\frac{\partial U_{n}}{\partial n}\right)_{e^{\prime}}=\frac{\left(U_{n}\right)_{e^{\prime}}-\left(U_{n}\right)_{P}}{L_{\left(e^{\prime}, P\right)}}
$$

where $e^{\prime}$ is the point of intersection of the line passing through node $P$ parallel to normal vector to $\Gamma^{1}$ at point " $e$ " and the line coincide with boundary $\Gamma^{1}$ (see Figure 2(b)). Following (3.28) and (3.29), the normal velocity component at point $e^{\prime}$ is approximated as

$$
\left(\vec{u}^{n+1} \cdot \vec{n}\right)_{e^{\prime}}=\left(U_{n}^{n+1}\right)_{e^{\prime}}=\left(U_{n}^{n+1}\right)_{P}-L_{\left(e^{\prime}, P\right)}\left(\frac{\partial G}{\partial \tau}\right)_{e^{\prime}}
$$

The discrete pressure equation for control volume $\Omega$ near flow-through boundary has the following form:

$\frac{1}{\Delta \Omega}\left[\left(\vec{u}^{n+1} \cdot \vec{n}\right)_{e^{\prime}} S_{e}+\sum_{c=s, w, n}\left(\vec{u}^{*} \cdot \vec{n}\right)_{c} S_{c}\right]-\frac{\Delta t}{\Delta \Omega} \sum_{c=s, w, n}\left(D_{n} p^{n+1}\right)_{c} S_{c}=0$.

Here, the point " $e$ "' is used instead of " $e$ " to approximate the flux through the east face. In this case the order of approximation is reduced to first order. Moreover, in 
many cases, the grid is arranged such that " $e^{\prime \prime \prime}$ coincides with the center of the east face. Substituting (3.30) into (3.31) and utilizing (3.13) at node $P$ yields

$$
\begin{aligned}
& \left.\frac{1}{\Delta \Omega}\left[\left(\vec{u}^{*} \cdot \vec{n}\right)_{P}-\Delta t\left(\nabla p^{n+1, s+1} \cdot \vec{n}\right)_{P}-L_{\left(e^{\prime}, P\right)} \frac{\partial G}{\partial \tau}\right]_{e^{\prime}}\right] S_{e} \\
& \quad+\frac{1}{\Delta \Omega}\left[\sum_{c=s, w, n}\left(\vec{u}^{*} \cdot \vec{n}\right)_{c} S_{c}\right]-\frac{\Delta t}{\Delta \Omega} \sum_{c=s, w, n}\left(D_{n} p^{n+1, s+1}\right)_{c} S_{c}=0 .
\end{aligned}
$$

The derivative of pressure with respect to outward normal direction $n$ at node $P$ approximated by one-side difference is

$$
\left(D_{n} p\right)_{P}^{n+1, s+1}=\frac{p_{e^{\prime}}^{n+1}-p_{P}^{n+1, s+1}}{L_{\left(P, e^{\prime}\right)}}
$$

where $L_{\left(P, e^{\prime}\right)}$ is the distance between nodes $P$ and $e^{\prime}$ on the boundary $\Gamma^{1}$.

(c) The tangential velocity and total pressure are prescribed (see, (2.4)) by

$$
(\vec{u} \cdot \vec{\tau})_{\Gamma^{1}}=G(x, y, t), \quad p+\frac{1}{2}|\vec{u} \cdot \vec{u}|=H(x, y, t), \quad(x, y) \in \Gamma^{1}
$$

When the tangential velocity and total pressure are specified on the flow-through part, the situation arises where mass flux, convective flux, and pressure are not known. Let us use a local coordinates system $(n, \tau)$ as in the previous case. The flux $\left(U_{n}\right)_{e^{\prime}}=(\vec{u} \cdot \vec{n})_{e^{\prime}}$ is approximated by (3.30). Since the pressure term on the flowthrough boundary $\Gamma^{1}$ (see Figure 2(b)) is unknown, one needs to approximate the pressure on the flow-through part by using the total pressure boundary condition, and one needs to calculate the pressure at point $e^{\prime}$. The total pressure on flowthrough part can be expressed in terms of local orthogonal coordinates $(n, \tau)$ in $2 D$ at point $e^{\prime}$ as

$$
p_{e^{\prime}}^{n+1, s+1}+\frac{1}{2}\left|\vec{U}_{e^{\prime}}^{n+1}\right|^{2}=p_{e^{\prime}}^{n+1, s+1}+\frac{1}{2}\left(\left(U_{n}^{n+1}\right)_{e^{\prime}}^{2}+\left(U_{\tau}\right)_{e^{\prime}}^{2}\right)=H
$$

Using boundary condition (3.34) the last equation recasts as

$$
p_{e^{\prime}}^{n+1, s+1}+\frac{1}{2}\left(U_{n}^{n+1}\right)_{e^{\prime}}^{2}=H-\frac{1}{2} G_{e^{\prime}}^{2} .
$$

Substituting $\left(U_{n}^{n+1}\right)_{e^{\prime}}$ given by (3.30) yields

$$
p_{e^{\prime}}^{n+1, s+1}+\frac{1}{2}\left(\left(U_{n}^{n+1}\right)_{P}-\frac{L_{\left(e^{\prime}, P\right)}}{2} \frac{\partial G}{\partial \tau}\right)^{2}=H-\frac{1}{2} G_{e^{\prime}}^{2} .
$$




$$
\begin{aligned}
& \text { Using }\left(U_{n}^{n+1}\right)_{P}=\left(\vec{u}^{n+1} \cdot \vec{n}\right)_{P}=\left(\vec{u}^{*} \cdot \vec{n}\right)_{P}-\Delta t\left(\nabla p^{n+1, s+1} \cdot \vec{n}\right)_{P} \text { yields } \\
& p_{e^{\prime}}^{n+1, s+1}+\frac{1}{2}\left[\left(\vec{u}^{*} \cdot \vec{n}\right)_{P}-\Delta t\left(\nabla p^{n+1, s+1} \cdot \vec{n}\right)_{P}-L_{\left(e^{\prime}, P\right)} \frac{\partial G}{\partial \tau}\right]^{2}=H-\frac{1}{2} G_{e^{\prime}} .
\end{aligned}
$$

Dropping terms of order $O(\Delta t)$, one gets

$$
\begin{aligned}
p_{e^{\prime}}^{n+1, s+1}= & H-\frac{1}{2} G_{e^{\prime}}-\frac{1}{2}\left(\vec{u}^{*} \cdot \vec{n}\right)_{P}^{2}-\frac{1}{2} L_{\left(e^{\prime}, P\right)}^{2}\left(\frac{\partial G}{\partial \tau}\right)_{e^{\prime}}^{2} \\
& +\left(\vec{u}^{*} \cdot \vec{n}\right)_{P} L_{\left(e^{\prime}, P\right)}\left(\frac{\partial G}{\partial \tau}\right)_{e^{\prime}}
\end{aligned}
$$

We have the previous case where pressure is given on the flow-through parts. When on the flow-through boundary $\vec{n}=\vec{\xi}$ and $G=0$, the expression for $p_{e}$ (3.38) reads

$$
p_{e}^{n+1, s+1}=H-\left(\vec{u}^{*} \cdot \vec{n}\right)_{P}^{2}
$$

\section{Results and Discussion}

The proposed method is applied to test problems. The details of each of the problems and computed results are discussed in the following sections.

\subsection{Flow between Two Parallel Plates}

The purpose of this test is to estimate the potential and quality of the developed method in the case of unsteady flow. Considering the $2 D$ channel flow between two parallel plates, the Cartesian coordinate system $(x, y, z)$ is chosen so that the $x$-axis is taken as the direction of flow, $y$ is the coordinate normal to the plate, and $z$ is the coordinate normal to $x$ and $y$, respectively. The velocity field is assumed to be of the form $\vec{u}=u(y, t) \vec{i}$, where $u$ is the velocity in the $x$-coordinate direction, and $\vec{i}$ is the unit vector in the $x$-coordinate direction. The Navier-Stokes equation implies that the pressure gradient is a function of time only, $\partial p / \partial x=f(t)$.

Initial data at $t=0$ is the fluid at the rest, $u(y, 0)=0$. The flow is driven by pressure difference $p_{2}(L, t)-p_{1}(0, t)=\Delta p \cos (\omega t)$ where $L$ is the distance between the flow-through parts, $\omega$ is the frequency, and $\Delta p$ is the characteristic pressure difference between two flowthrough parts. The problem is dimensionalized with the height of the channel $h$ as the length scale, $\Delta p \cdot h / L$ as the pressure scale, $\sqrt{\Delta p \cdot h} / \sqrt{\rho L}$ as the velocity scale, and $\sqrt{\rho h L} / \sqrt{\Delta p}$ as the time scale. Nondimensional frequency is $\eta=\omega \sqrt{\Delta p} / \sqrt{\rho L h}$. Since the flow is driven by pressure difference and there is no velocity scale in the problem, we use $\rho U^{2}=\Delta p \cdot h / L$ in the traditional definition of the Reynolds number and call it the "Pressure Reynolds Number."

$$
\operatorname{Re}_{\Delta p}=\frac{U h}{v}=\frac{h}{v} \sqrt{\frac{\Delta p h}{\rho L}}
$$




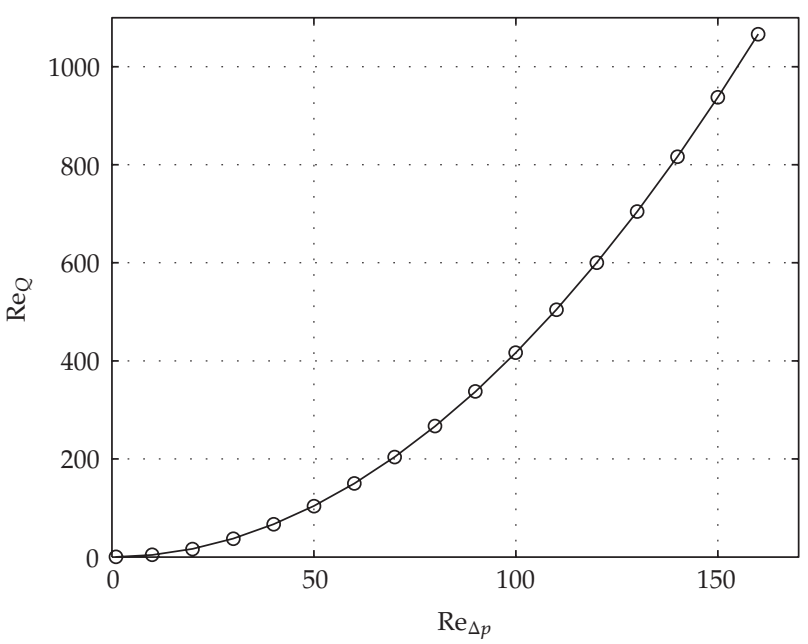

Figure 3: The relation between $\operatorname{Re}_{Q}$ and $\operatorname{Re}_{\Delta p}$ for $\eta=0$.

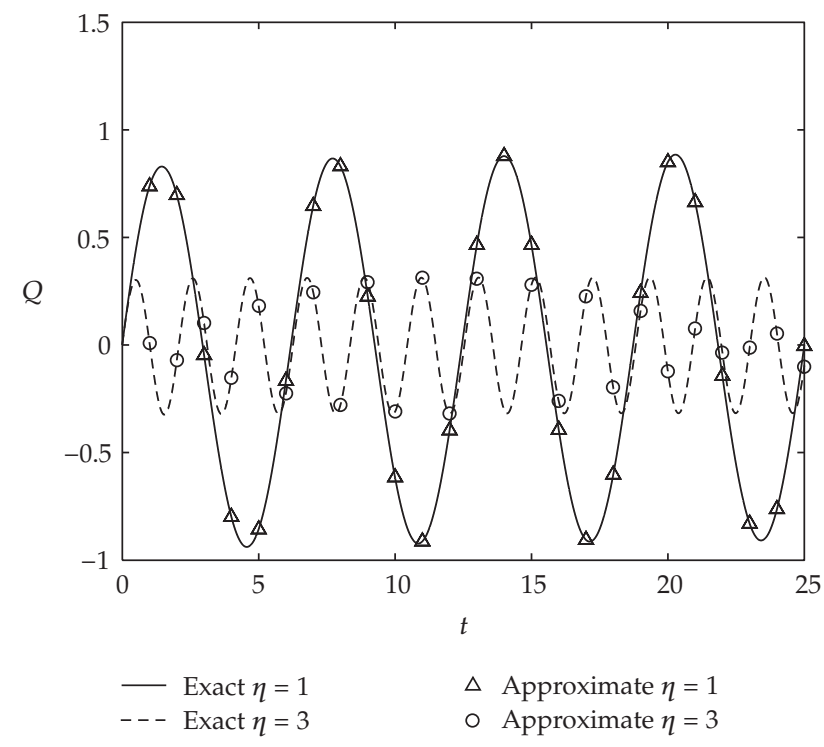

Figure 4: Volume rate versus time.

where $v$ is the kinematic viscosity. The analytical solution of the dimensionless problem obtained by separation of variables is

$$
u(y, t)=\sum_{n=1}^{\infty}\left[\frac{2(1-\cos (n \pi))}{n \pi} \sin (n \pi y) \int_{0}^{t} e^{-\lambda_{n}^{2}(t-\tau)} \cdot \cos (\eta \tau) d \tau\right], \quad \lambda_{n}=\frac{n \pi}{\operatorname{Re}_{\Delta p}^{1 / 2}}, 0 \leq y \leq 1
$$

Computations are carried out with 1000 cells distributed in a uniform manner in the channel. A uniform grid having 20 lines across the channel and 50 lines in the direction of $x$ was found 
to reproduce the flow parameters with good accuracy. In order to reduce computing cost, the distance between the flow-through parts was chosen to be one, $L=1$. The dependence between $\operatorname{Re}_{Q}$ and $\operatorname{Re}_{\Delta p}$ is plotted in Figure 3, for constant pressure drop $p_{2}(L, t)-p_{1}(0, t)=$ $\Delta p$. The solid line represents the exact relation $\operatorname{Re}_{Q}=\operatorname{Re}_{\Delta p}^{2} / 24$, where $\operatorname{Re}_{Q}=Q / 2 v$ is the

Reynolds number based on the flow rate, $Q=\int_{0}^{1} u(y) d y$. Circle signs represent the results of our numerical simulations. The Reynolds number $\operatorname{Re}_{Q}$ is not known a priori; it was computed at the end of the numerical simulation from the steady state flow rate obtained with the given $\operatorname{Re}_{\Delta p}$. As expected, the results are very close, and the velocity profile for all cases was the parabolic Poiseuille flow.

From the analytical solution given by (4.2), it is obvious that the mass flow rate oscillation is a function of the oscillating frequency $\eta$ and the pressure Reynolds number, $\operatorname{Re}_{\Delta p}$. In Figure 4, the variation of $Q(t)=\int_{0}^{1} u(y, t) d y$ with time is shown for given $\eta=1$ and 3, and $\operatorname{Re}_{\Delta p}=150$. Solid and dashed lines represent exact solutions for $\eta=1$ and 3, respectively. Circle and triangle signs correspond to the result of our numerical simulations for $\eta=1$ and 3 , respectively. The numerical solution starts at $t=0$, and the time step is $\Delta t=10^{-4}$. The above result corroborates that the proposed numerical method successfully predicts the volume rate for the constant and oscillated pressure drop.

\subsection{Flow with Circular Streamline}

Another simple type of fluid motion through a bounded domain is one in which all the streamlines are circles centered on a common axis of symmetry. Steady motion can be generated by a circumferential pressure gradient in the domain between two concentric cylinders of radii $r_{1}$ and $r_{2}$. If the motion is to remain purely rotatory with the axial component of velocity to be zero, the axial pressure gradient must be zero, and the Navier-Stokes equations show that motion must be $2 D$. Using the equation of motion in polar coordinates $(r, \theta)$ and assuming that the velocity component in direction of the $\theta$-coordinate line $v=v(r)$ is a function of $r$ only, and the radial velocity component is zero, one finds

$$
\begin{gathered}
\frac{v^{2}}{r}=\frac{\partial p}{\partial r} \\
\frac{\partial^{2} v}{\partial r^{2}}+\frac{1}{r} \frac{\partial v}{\partial r}-\frac{v}{r^{2}}=\frac{1}{r} \frac{\partial p}{\partial \theta} .
\end{gathered}
$$

The variables in (4.3) are made nondimensional with $h=r_{2}-r_{1}$ as length scale, $v / h$ as velocity scale, and $\rho v^{2} / h^{2}$ as pressure scale. Let $d_{0}=\left(r_{1}+r_{2}\right) /(2 h)=R_{0} / h$ be the nondimensional radius of centerline. Figure 5 represents a sketch of the problem geometry and main notations. It is easy to see from (4.3) that pressure has to be a linear function of $\theta$ :

$$
p(r, \theta)=f(r)+K \cdot \theta, \quad K=\frac{\partial p}{\partial \theta}-\text { const, } f(r)=\int_{d_{0}-1 / 2}^{r} \frac{v^{2}(\xi)}{\xi} d \xi
$$

With the boundary condition

$$
v\left(d_{0} \pm \frac{1}{2}\right)=0
$$




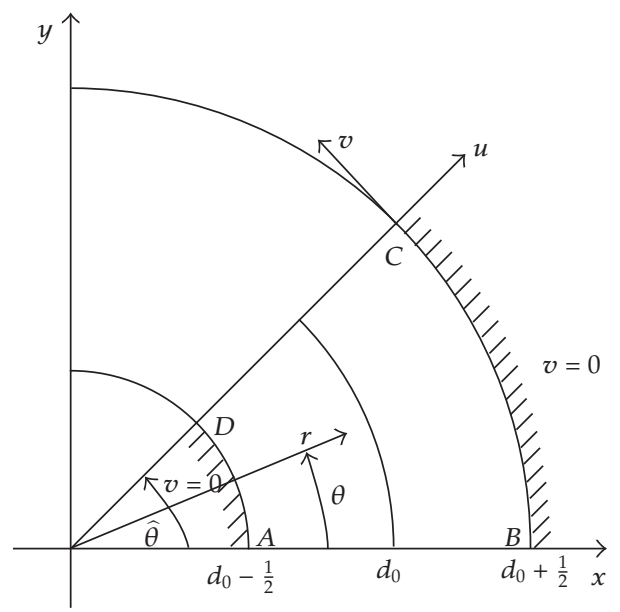

Figure 5: The sketch of problem domain, flow with circular streamlines.

one obtains solution of (4.3)-(4.5) in the following form:

$$
\begin{gathered}
v(r)=\frac{K}{8}\left\{C_{1} r+\frac{C_{2}}{r}+4 r \ln r\right\}, \quad d_{0}-\frac{1}{2} \leq r \leq d_{0}+\frac{1}{2} \\
p(r, \theta)=\int_{d_{0}-1 / 2}^{r} \frac{v^{2}(\xi)}{\xi} d \xi+K \cdot \theta, \quad 0 \leq \theta \leq \widehat{\theta}, d_{0}-\frac{1}{2} \leq r \leq d_{0}+\frac{1}{2} \\
C_{1}=\frac{\left(2 d_{0}-1\right)^{2} \ln \left(d_{0}-1 / 2\right)-\left(2 d_{0}+1\right)^{2} \ln \left(d_{0}+1 / 2\right)}{2 d_{0}}, \quad C_{2}=\frac{\left(4 d_{0}^{2}-1\right)^{2}}{8 d_{0}} \ln \left(\frac{d_{0}+1 / 2}{d_{0}-1 / 2}\right) .
\end{gathered}
$$

The nondimensional volume rate of flow becomes

$$
\begin{aligned}
& Q=\int_{d_{0}-1 / 2}^{d_{0}+1 / 2} v d r=\frac{K}{8} E \\
& E=2 C_{1} d_{0}+C_{2} \ln \left(\frac{\left(d_{0}+1 / 2\right)}{\left(d_{0}-1 / 2\right)}\right)-4 d_{0}+2\left[\left(d_{0}+\frac{1}{2}\right)^{2} \ln \left(d_{0}+\frac{1}{2}\right)-\left(d_{0}-\frac{1}{2}\right)^{2} \ln \left(d_{0}-\frac{1}{2}\right)\right] .
\end{aligned}
$$

Problem (4.3)-(4.5) can be considered as an example of the flowing-through problem where pressure and the tangential component of the velocity vector are given on flow-through parts $A B$ and $D C$. It is worth to note here that the distribution of pressure is not constant at the flow-through parts and that the numerical solution uses the Navier-Stokes equation in terms of Cartesian coordinates and Cartesian velocity components $\vec{u}=\left(u_{x}, u_{y}\right)$ where $u_{x}=-v(r) \sin (\theta), u_{y}=v(r) \cos (\theta), r^{2}=x^{2}+y^{2}$, and $\theta=\tan ^{-1}(y / x)$. Using exact solution (4.6)-(4.9), one can formulate the flowing-through problem where total pressure $p+(1 / 2)\left[u_{x}^{2}(r, \theta)+u_{y}^{2}(r, \theta)\right]$ and tangent velocity are known on flow-through parts. It is also possible to consider problems where, in flow-through parts, different kinds of boundary 


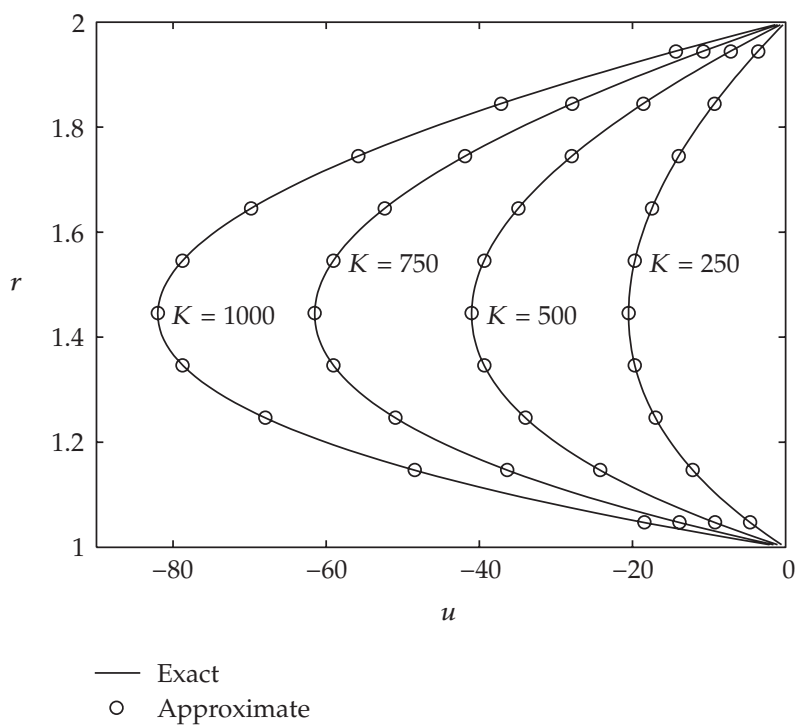

Figure 6: Velocity profiles at the verticle line $\theta=\pi / 2$ (component $u_{x}$ ), for Case 1 and different values of $K$.

Table 1: Test cases $0 \leq \theta \leq \pi, \vec{u}=\left(u_{x}, u_{y}\right)$, and $H(r)=p+v(r)^{2} / 2$.

\begin{tabular}{lccc}
\hline Case & \multicolumn{2}{c}{ Flow-through boundary } & Solid wall \\
& $A B(\theta=0)$ & $C D(\theta=\pi)$ & $A D, C B(0 \leq \theta \leq \pi)$ \\
\hline 1 & $u_{x}=0, p=f(r)$ & $u_{x}=0, p=f(r)+K \pi$ & $u_{x}=u_{y}=0$ \\
2 & $u_{x}=0, u_{y}=v(r)$ & $u_{x}=0, p=f(r)+K \pi$ & $u_{x}=u_{y}=0$ \\
3 & $u_{x}=0, u_{y}=v(r)$ & $u_{x}=0, p+u_{y}^{2} / 2=H(r)$ & $u_{x}=u_{y}=0$ \\
4 & $u_{x}=0, p=f(r)$ & $u_{x}=0, p+u_{y}^{2} / 2=H(r)$ & $u_{x}=u_{y}=0$ \\
\hline
\end{tabular}

conditions apply. The test cases of flowing-through problems computed in this section are summarized in Table 1 . In all cases, we use $0 \leq \theta \leq \pi$. Nonorthogonal logically rectangular boundary-fitted grids were constructed as follows. The impermeable boundaries $A D$ and $C B$ are partitioned equally into $M$ subintervals. The flowing-though parts $A B$ and $C D$ are divided into an equal number of $N$ subintervals. To reach steady flow, we used marching in time until the solution no longer changes. The grid independence study has been carried out for several values of circumferential pressure gradient, $K$, and for four cases of the flowingthrough problems. The influence of the grid size on the difference between the exact velocity (4.6) and the approximate velocity in the maximum norm is shown in Table 2, for $K=500$. The convergence rates for the two finest grids are compared to the next coarser grid (see values in the brackets). Upper indices "ext" and "app" reference the exact and approximate solutions, respectively. It can be clearly seen from these results that the rate of convergence is near two. For Case 1, Figure 6 shows the variation of the dimensionless $x$-component of the velocity vector along the line $\theta=\pi / 2$ with circumferential pressure gradient $\partial p / \partial \theta=K$. The value of the circumferential pressure gradient varies from $K=250$ to $K=1000$.

Figure 7 shows pressure distribution for Case 1 along the line $\theta=\pi / 2$ and $K=500$. In both figures the solid lines represent the exact solutions (4.6) and (4.7), and the circle signs represent the numerical results. The calculated velocity profile and pressure along the line $\theta=$ const for Cases $2-4$ are also in excellent agreement with the exact solution. 


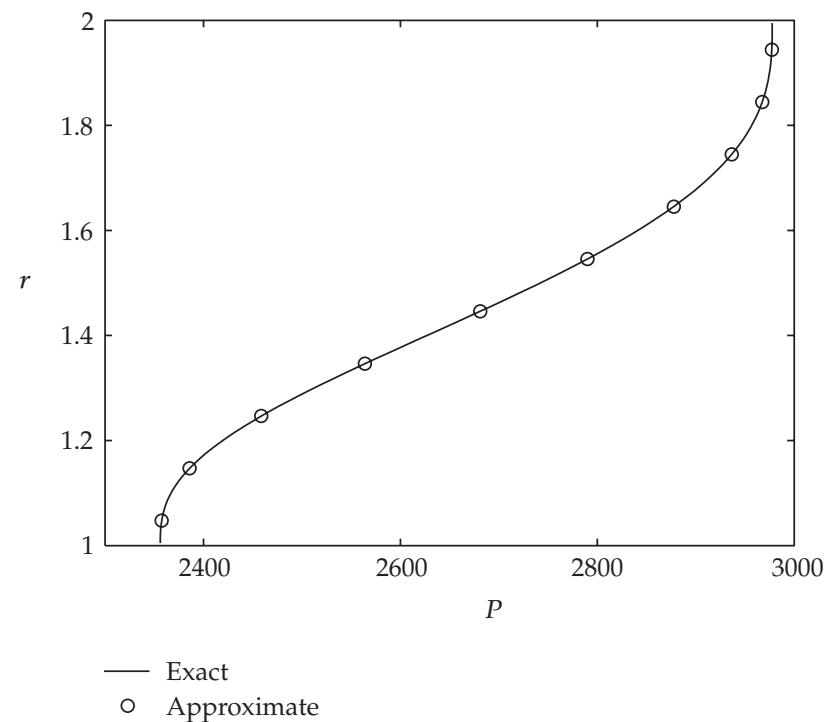

Figure 7: Pressure along the line $\theta=\pi / 2$ for Case 1 .

Table 2: Rate of convergence of $u_{y}$ for test cases.

\begin{tabular}{lcccc}
\hline \multirow{2}{*}{ Grid $M \times N$} & \multicolumn{4}{c}{$\left\|u^{\text {app }}-u^{\text {ext }}\right\|$} \\
& Case 1 & Case 2 & Case 3 & Case 4 \\
\hline $20 \times 10$ & $1.508 \mathrm{E}-1$ & $1.220 \mathrm{E}-1$ & $1.218 \mathrm{E}-1$ & $1.425 \mathrm{E}-1$ \\
$40 \times 20$ & $3.979 \mathrm{E}-2(1.92)$ & $3.026 \mathrm{E}-2(2.01)$ & $3.018 \mathrm{E}-2(2.01)$ & $3.753 \mathrm{E}-2(1.93)$ \\
$80 \times 40$ & $9.955 \mathrm{E}-3(1.99)$ & $8.291 \mathrm{E}-3(1.87)$ & $7.590 \mathrm{E}-3(1.99)$ & $9.739 \mathrm{E}-3(1.95)$ \\
\hline
\end{tabular}

\subsection{Flowing-Through Problem for U-Bend Channel}

For further validation, two-dimensional U-bend channel flow simulations are conducted. The flow configuration and main notations are shown in Figure 8. The channel has a curvature ratio $\delta=R / d$, where $R$ is the radius of curvature, and $d$ is the width of channel. The lengths of the channel before and after the bend $L$ are taken sufficiently large to assume that pressure at sections $A_{1} A_{1}^{\prime}$ and $A_{2} A_{2}^{\prime}$ can be considered as constant, and fluid enters or leaves the channel legs with laminar, fully developed velocity profiles. The developed finite volume method has been utilized to simulate steady flow. For obtaining steady-state solution, the time is considered as pseudotime, and equations are iterated until the solution converges to steady state. Three kinds of the flowing-through problem have been considered. In all cases, no-slip boundary condition holds at the impermeable parts $\Gamma_{1}^{0}$ and $\Gamma_{2}^{0}$.

The three flowing-through problems are formulated as follows.

(P1) On flow-through parts $\Gamma_{1}^{1}$ and $\Gamma_{2}^{1}$, the tangent components of velocity vector and pressure are specified (see (2.5)) by

$$
\begin{array}{ll}
\vec{u} \cdot \vec{\tau}=u_{x}=0, & p=p_{1}, \quad(\vec{x}) \in \Gamma_{1}^{1}, \\
\vec{u} \cdot \vec{\tau}=u_{x}=0, & p=p_{2}, \quad(\vec{x}) \in \Gamma_{2}^{1},
\end{array}
$$

where $\vec{\tau}$ is tangent unit vector to $\Gamma_{1}^{1}$ and $\Gamma_{2}^{1}$. 


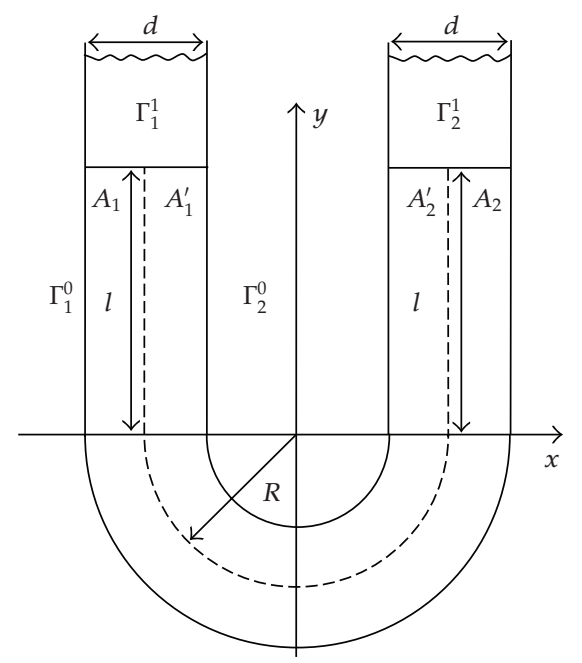

Figure 8: Schematic diagram of U-bend channel.

(P2) On flow-through part $\Gamma_{1}^{1}$, the tangent and normal components of velocity vector are given (see (2.6)) by

$$
\vec{u}=\left(u_{x}, u_{y}\right)=\left(0, u_{s}^{1}(x)\right), \quad \vec{x} \in \Gamma_{1}^{1}
$$

where $u_{s}^{1}(x)$ is the parabolic Poiseuille velocity profile.

On flow-through part $\Gamma_{2}^{1}$, the tangent component of velocity and pressure are specified (see (2.5)) by

$$
\vec{u} \cdot \vec{\tau}=u_{x}=0, \quad p=p_{2}, \quad(\vec{x}) \in \Gamma_{1}^{1}
$$

(P3) On flow-through part $\Gamma_{1}^{1}$, the tangent component of velocity vector and total pressure are prescribed (see (2.4)) by

$$
\vec{u} \cdot \vec{\tau}=u_{x}=0, \quad p+\frac{1}{2} \rho|\vec{u} \cdot \vec{u}|=H_{1}(\vec{x}), \quad(\vec{x}) \in \Gamma_{1}^{1}
$$

where $H_{1}(\vec{x})$ is a given function and is computed from the solution of P2. On the flow-through parts $\Gamma_{2}^{1}$, the tangent component of the velocity vector and pressure are known (see (2.5)) by

$$
\vec{u} \cdot \vec{\tau}=u_{x}=0, \quad p=p_{2}, \quad(\vec{x}) \in \Gamma_{2}^{1}
$$




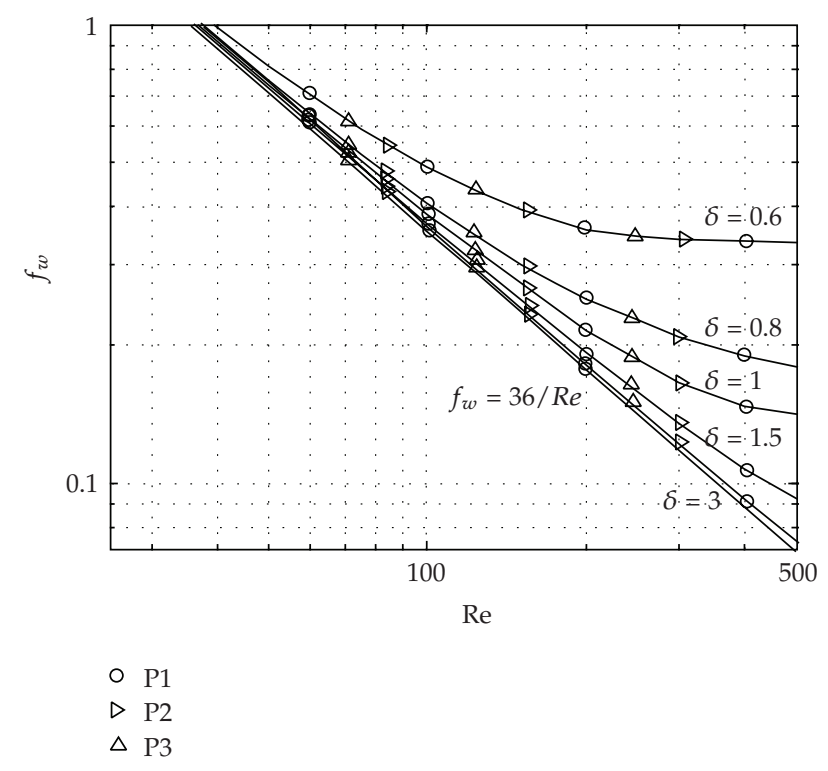

Figure 9: Friction factor as a function of the Reynolds number.

The main characteristic of flow in curve channels is pressure loss. The pressure losses are presented in the form of friction factor versus Reynolds number:

$$
f_{w}=\frac{2 \Delta p}{\rho U^{2}(L / d)}=f(\operatorname{Re})
$$

where $U$ is the mean velocity, $\rho$ is the density of the fluid, $\operatorname{Re}=U d / v$ is the Reynolds number, $v$ is the kinematic viscosity of fluid, and $\Delta p$ is the pressure losses, $\Delta p=p_{2}-p_{1}$. Before the main computations were started, a test was executed with a straight channel. A very good agreement of the computed pressure losses with the theoretical solution based on the Poiseuille law $f_{w} \approx 36 /$ Re was observed. Based on the preliminary experiments, the length of the channel legs $l=L / d=5$ was used in the main computations represented below. The impermeable boundaries $A_{1} A_{2}$ and $A_{1}^{\prime} A_{2}^{\prime}$ were equally partitioned into $M$ subintervals. The flowing-through parts $A_{1} A_{1}^{\prime}$ and $A_{2} A_{2}^{\prime}$ were divided into an equal number of $N$ subintervals. Three grid sequences of $100 \times 10,200 \times 20$, and $400 \times 40$ nodes were tested. Computations using these grid sequences are shown in Table 3 . In the case of the flowing-through problem P1, the pressure losses are known a priori, and the Reynolds number was computed from the steady state flow rate. In problem P2 the Reynolds number is known a priori, and $\Delta p$ was estimated from the steady state flow regime. In the problem P3 neither $\Delta p$ nor Re is known a priori, and both of them were computed at the end of the numerical simulation from steady state.

Total pressure losses of a U-bend channel flow are presented in the form of the friction factor versus Reynolds number $f_{w}=f(\operatorname{Re})$ in Figure 9, where the effect of the dimensionless curvature ratio, $\delta=R / d$, is shown. All three flowing-through problems P1, P2, and P3 give very close results. From Figure 9 it is seen that the effect of the channel curvature ratio on the friction factor is small for $\delta>3$ for all tested flowing-through problems. The friction factor $f_{w}$ increases with decreasing $\delta$. In Figure 10, streamline patterns are presented. Figure 10(a) is drawn for $\delta=1$ and $\operatorname{Re}=200$, Figure 10(b) shows the case of $\delta=1$ and $\operatorname{Re}=300$, Figure 10(c) 
Table 3: Friction factor for three kinds of the flowing-through problem. $\operatorname{Re}=100, \delta=3$.

\begin{tabular}{cccc}
\hline Grid $M \times N$ & & Friction factor, $f_{w}$ & P3 \\
\hline $100 \times 10$ & P1 & P2 & 0.431782 \\
$200 \times 20$ & 0.43128 & 0.433153 & 0.429832 \\
$400 \times 40$ & 0.429673 & 0.431639 & 0.429587 \\
\hline
\end{tabular}

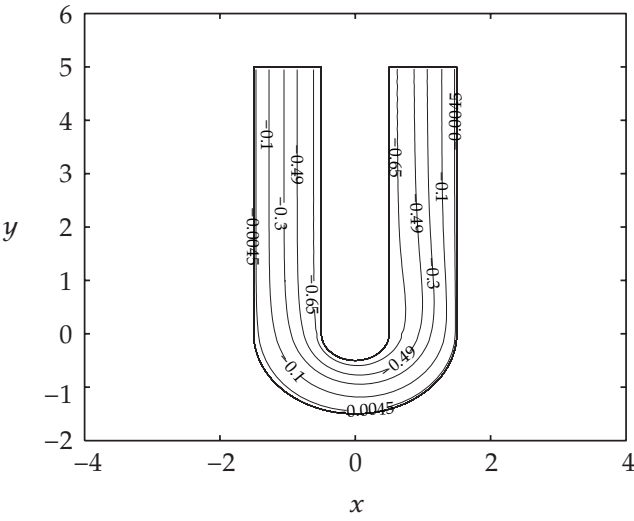

(a) $\delta=1.0, \operatorname{Re}=200$

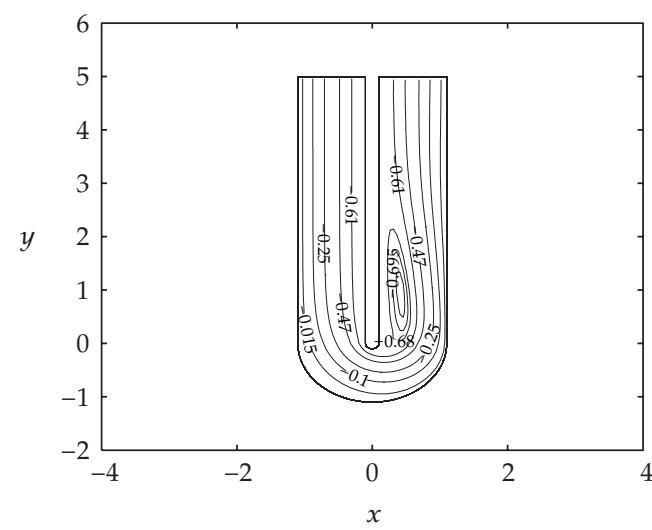

(c) $\delta=0.6, \operatorname{Re}=200$

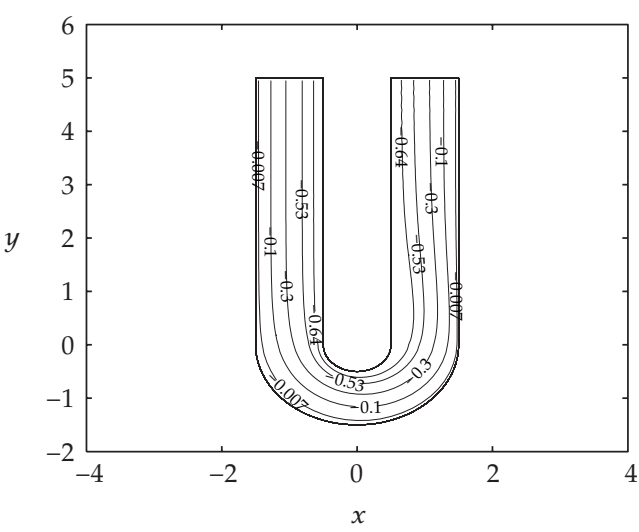

(b) $\delta=1.0, \operatorname{Re}=300$

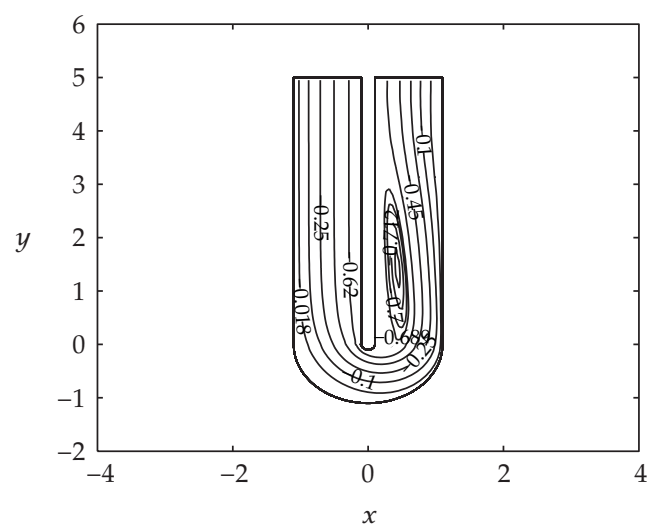

(d) $\delta=0.6, R e=300$

Figure 10: Streamline patterns of flow in the U-bend channel for various $\delta$ and Re.

depicts $\delta=0.6$ and $\operatorname{Re}=200$, and Figure 10(d) is drawn for $\delta=0.6$ and $\operatorname{Re}=300$. The sharp bend $\delta=0.6$ and increasing Reynolds number cause separation which occurs on the right side of the bend. The size of the separation zone increases with increasing flow rate and decreasing $\delta$.

The velocity profile in the cross section $y=1$ of the right-hand side leg of U-bend is depicted in Figure 11 for $\operatorname{Re}=200$ and 300 and $\delta=0.6$.

\subsection{Flow in Planar T-Junction Channel}

The T-junction flow geometry is schematically represented in Figure 12. The origin of the coordinate system is located in the lower horizontal boundary opposite the left corner of 


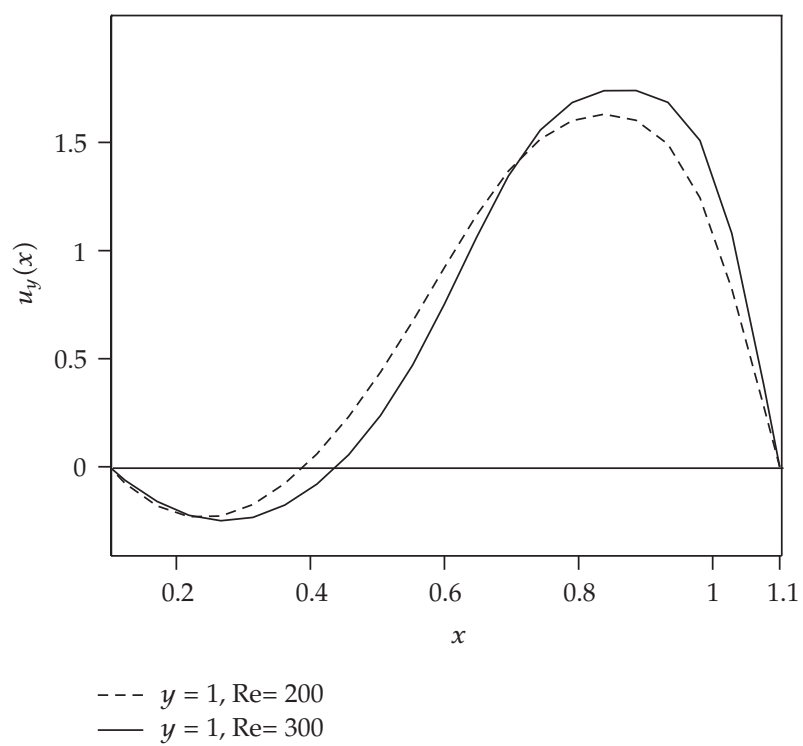

Figure 11: Velocity profile in section $y=1$ of right-hand side of U-bend channel. $\delta=0.6, \operatorname{Re}=200$ and 300 .

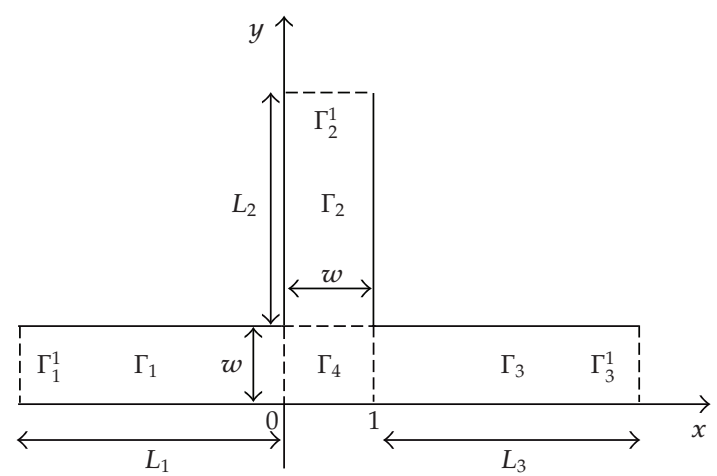

Figure 12: Schematic geometry of T-junction bifurcation and coordinate system.

branch as demonstrated. The left-hand side branch, the upper branch, the right-hand side branch, and the junction area are denoted by $\Gamma_{1}, \Gamma_{2}, \Gamma_{3}$, and $\Gamma_{4}$, respectively. All branches have the same width $w$.

The flow rate ratio is defined as $\beta \equiv Q_{3} / Q_{1}$ where $Q_{1}$ and $Q_{3}$ are the inlet duct and branch duct flow rates per unit span, respectively. The following problem has been considered.

(i) On flow-through part $\Gamma_{1}^{1}$ a laminar, fully developed, parabolic velocity profile is prescribed by

$$
\vec{u}=\left(u_{x}(y), 0\right), \quad(x, y) \in \Gamma_{1}^{1} .
$$




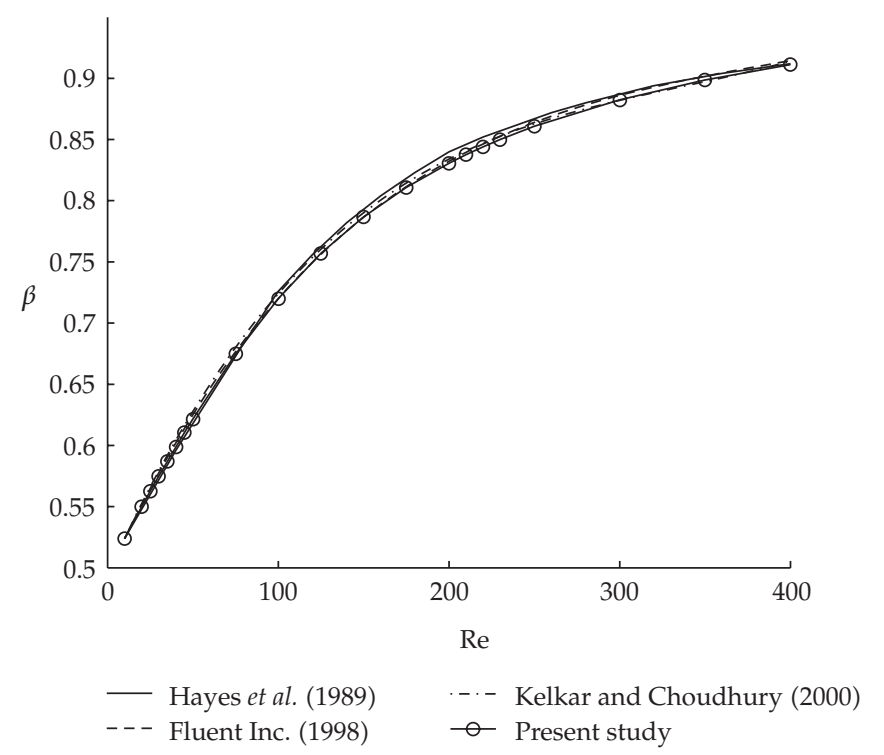

Figure 13: The flow rate ratio, $\beta$, as a function of Reynolds number, Re.

(ii) On flow-through parts $\Gamma_{2}^{1}$ and $\Gamma_{3}^{1}$ the tangent component of the velocity vector and the pressure are specified by

$$
\begin{array}{lll}
\vec{u} \cdot \vec{\tau}_{2}=u_{y}=0, & p=p_{2}, & (x, y) \in \Gamma_{2}^{1}, \\
\vec{u} \cdot \vec{\tau}_{3}=u_{x}=0, & p=p_{3}, & (x, y) \in \Gamma_{3}^{1},
\end{array}
$$

where $\vec{\tau}_{i}$ is the unit tangent vector to $\Gamma_{i}^{1}, i=2,3$.

The calculations are compared with those of Hayes et al. [13], Kelkar and Choudhury [16], and Fluent [24]. A flowing-through problem with $u_{x}=4 y-4 y^{2}$ and equal static pressure $p_{2}=p_{3}=0$ is considered. The Navier-Stokes equation dimensionalized with the width, $w$, as characteristic length, the inlet centerline velocity $U_{c}$ as the characteristic velocity, and $\rho U_{c}^{2}$ as the scale of pressure. A range of Reynolds number $\operatorname{Re}=w U_{c} / v$, where $v$ is the kinematic viscosity, is studied with $\operatorname{Re} \in[10,400]$. The computational domain is set to have lengths of $L_{1} / w=2$ and $L_{2} / w=L_{3} / w=3$ according to the results represented in Fluent Inc. [24]. The square meshes containing 20,30, and 40 cells from wall to wall are used. The studied cases start from a motionless state. A steady flow is achieved if the following condition is held: $\left\|\vec{u}^{n+1}-\vec{u}^{n}\right\| \leq \varepsilon=10^{-8}$. The maximum norm of grid function is used. Figure 13 shows the effect of increasing the Reynolds number on the flow split between the main and the side exit branches. The value of $\beta$ increases from 0.5 for a small Reynolds number, $\operatorname{Re}<10$, to about 0.9 at $\operatorname{Re}=400$. Figure 14 shows the predicted streamline pattern and pressure contour plots for two Reynolds Numbers $\operatorname{Re}=100,400$. Flow separation from the left wall of the upper branch occurs at all considered Reynolds numbers. These are very similar to those reported in Fluent Inc. (1998). The size and extent of flow separation zone are in a good agreement with results of Hayes et al. [13], Kelkar and Choudhury [16], and Fluent [24]. 


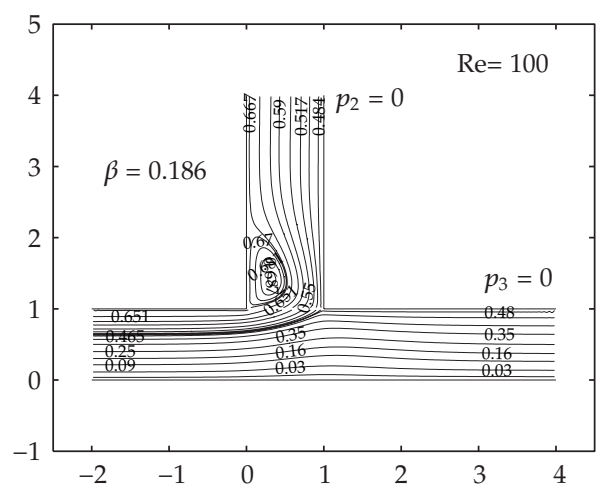

(a)

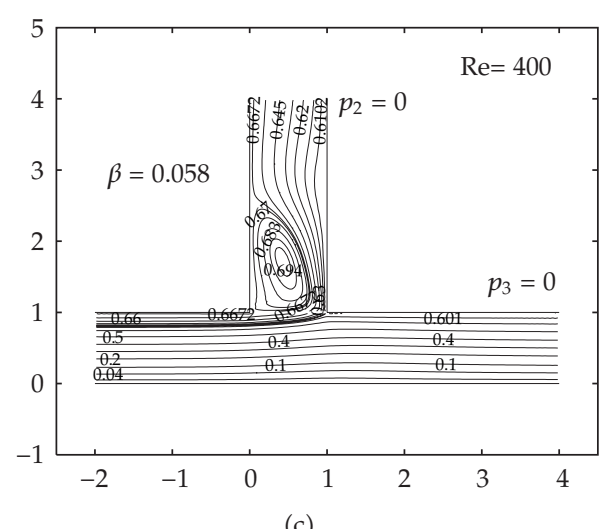

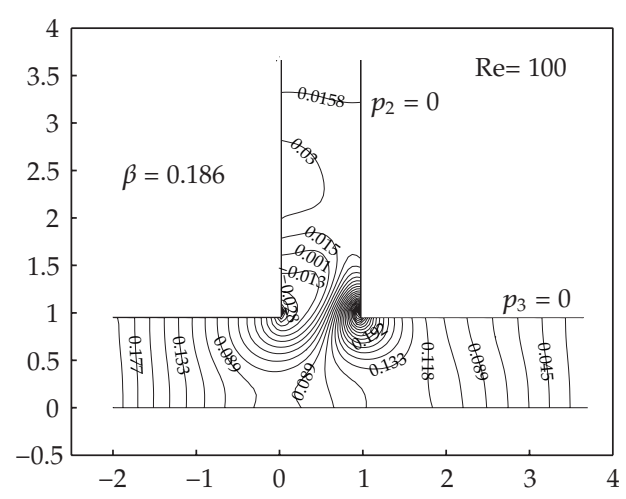

(b)

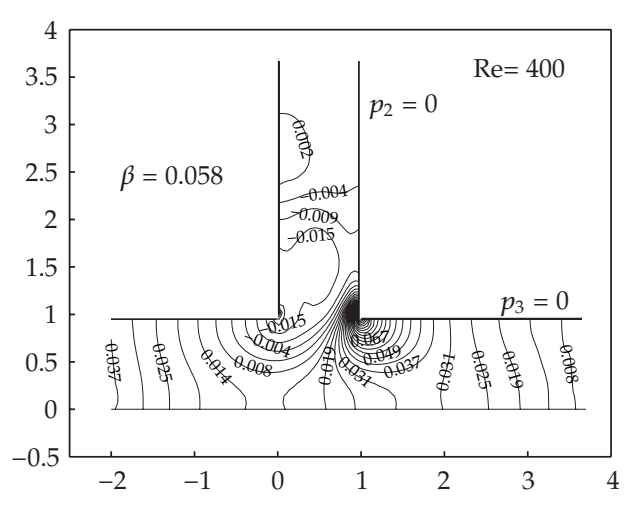

(d)

Figure 14: Streamline patterns and pressure contour of flow in the T-junction for various Re and equal static pressure at the exists $\Gamma_{2}^{1}$ and $\Gamma_{3}^{1}$.

\section{Conclusion}

A mathematical formulation of well-posed initial boundary value problems for viscous incompressible fluid flow-through-bounded domain is described for the case where the values of static or total pressure and tangential components of the velocity vector on flowthrough parts of the domain boundary are prescribed. A computational method for the approximate solution of these well-posed problems is developed within the framework of the finite volume approach. The robustness of the method is validated by its application for channel flows driven by pressure drop for which analytical solutions are available $(2 D$ Poiseuille flow, purely rotatory flow in the annular domain between cylinders). The effect of curvature ratio of planar U-bend channel is analyzed for various flowing-through problem formulations. The flow through planar T-junction channel is utilized as a benchmark test in the case of several flow-through parts of boundary. Results of all tests confirm the reliability and accuracy of developed method. The method is robust and accurate in simulating incompressible flows in domains with known boundary pressure (or total pressure) and with known velocity profiles in flow-through parts of boundary. 


\section{Acknowledgment}

This work was supported by the Royal Golden Jubilee Ph.D. Program (Contract no. PHD/0006/2548).

\section{References}

[1] S. N. Antontsev, A. V. Kazhikhov, and V. N. Monakhov, Boundary Value Problems in Mechanics of Nonhomogeneous Fluids, vol. 22 of Studies in Mathematics and Its Applications, North-Holland, Amsterdam, The Netherlands, 1990.

[2] H. Koch and D. Tataru, "Well-posedness for the Navier-Stokes equations," Advances in Mathematics, vol. 157, no. 1, pp. 22-35, 2001.

[3] O. A. Ladyzhenskaya, "Mathematical analysis of Navier-Stokes equation of incompressible liquids," Annual Review of Fluid Mechanics, vol. 7, pp. 249-272, 1963.

[4] V. V. Ragulin, "On the problem of a viscous fluid flowing through a bounded domain in the case of prescribed drop in pressure or heat," Dinamika Splošnoŭ Sredy, vol. 27, pp. 78-92, 1976.

[5] V. V. Ragulin and Sh. Smagulov, "Smoothness of the solution of a boundary value problem for NavierStokes equations," Chislennye Metody Mekhaniki Sploshnoı̆ Sredy, vol. 11, no. 4 inam, pp. 113-121, 1980.

[6] R. Temam, Navier-Stokes Equation: Theory and Numerical Analysis, Mir, Moscow, Russia, 1981.

[7] N. A. Petersson, "Stability of pressure boundary conditions for Stokes and Navier-Stokes equations," Journal of Computational Physics, vol. 172, no. 1, pp. 40-70, 2001.

[8] R. L. Sani, J. Shen, O. Pironneau, and P. M. Gresho, "Pressure boundary condition for the timedependent incompressible Navier-Stokes equations," International Journal for Numerical Methods in Fluids, vol. 50, no. 6, pp. 673-682, 2006.

[9] B. G. Kuznetsov, N. P. Moshkin, and Sh. Smagulov, "Numerical investigation of the flow of a viscous incompressible fluid in channels of complex geometry with specification of pressure drops," Chislennye Metody Mekhaniki Sploshnoŭ Sredy, vol. 14, no. 5, pp. 87-99, 1983.

[10] N. P. Moshkin, "Numerical simulation of viscous incompressible flow in channel under pre-assigned pressure drops," in Chislennye Metody Dinamiki Vyazkoi Zidkosti. Trudy IX Vsesouznoi Shkoly-Seminara, pp. 50-54, Institute of Theoretical and Applied Mechanics, Novosibirsk, Russia, 1983.

[11] N. P. Moshkin, "A method for the numerical solution of a flow problem in "streamfunction-vortex" variables," Chislennye Metody Mekhaniki Sploshnoı̆ Sredy, vol. 15, no. 3, pp. 98-114, 1984.

[12] N. P. Moshkin, "Numerical simulation of nonstationary viscous fluid flow with reassigned pressure drops," in Proceedings of the 4th International Conference on Boundary and Interior Layers (BAIL '86), Novosibirsk, Russia, July 1986.

[13] R. E. Hayes, K. Nandakumar, and H. Nasr-El-Din, "Steady laminar flow in a 90 degree planar branch," Computers \& Fluids, vol. 17, no. 4, pp. 537-553, 1989.

[14] M. H. Kobayashi, J. C. F. Pereira, and J. M. M. Sousa, "Comparison of several open boundary numerical treatments for laminar recirculating flow," International Journal for Numerical Methods in Fluids, vol. 16, no. 5, pp. 403-419, 1993.

[15] J. G. Heywood, R. Rannacher, and S. Turek, "Artificial boundaries and flux and pressure conditions for the incompressible Navier-Stokes equations," International Journal for Numerical Methods in Fluids, vol. 22, no. 5, pp. 325-352, 1996.

[16] K. M. Kelkar and D. Choudhury, "Numerical method for the prediction of incompressible flow and heat transfer in domains with specified pressure boundary conditions," Numerical Heat Transfer, Part B, vol. 38, no. 1, pp. 15-36, 2000.

[17] R. Fernandez-Feria and E. Sanmiguel-Rojas, "An explicit projection method for solving incompressible flows driven by a pressure difference," Computers \& Fluids, vol. 33, no. 3, pp. 463-483, 2004.

[18] W. L. Barth and G. F. Carey, "On a boundary condition for pressure-driven laminar flow of incompressible fluids," International Journal for Numerical Methods in Fluids, vol. 54, no. 11, pp. 13131325, 2007.

[19] A. J. Chorin and J. E. Marsden, A Mathematical Introduction to Fluid Mechanics, vol. 4 of Texts in Applied Mathematics, Springer, New York, NY, USA, 2nd edition, 1990.

[20] J. L. Guermond, P. Minev, and J. Shen, "An overview of projection methods for incompressible flows," Computer Methods in Applied Mechanics and Engineering, vol. 195, no. 44-47, pp. 6011-6045, 2006.

[21] R. Peyret and T. D. Taylor, Computational Methods for Fluid Flow, Springer Series in Computational Physics, Springer, New York, NY, USA, 1983. 
[22] D. Shepard, "A two-dimensional interpolation function for irregularly- spaced data," in Proceedings of the 23rd ACM National Conference, pp. 517-524, New York, NY, USA, January 1968.

[23] S. Muzaferija, Adaptive finite volume method for flow predictions using unstructured meshes and multigrid approach, Ph.D. thesis, University of London, London, UK, 1994.

[24] Fluent Inc., "FLUENT 5.0 UDF User's Guide," Fluent Incorporated Centerra Resource Park 10 Cavendish Court Lebanon. NH 03766, 1998. 


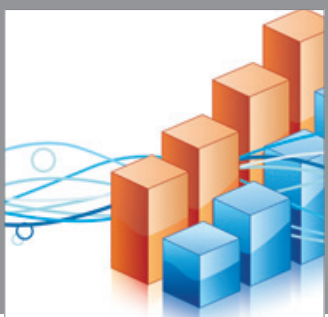

Advances in

Operations Research

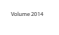

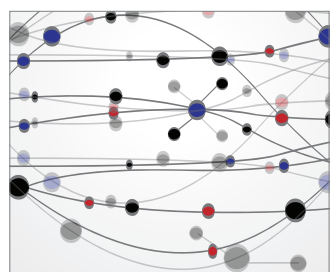

\section{The Scientific} World Journal
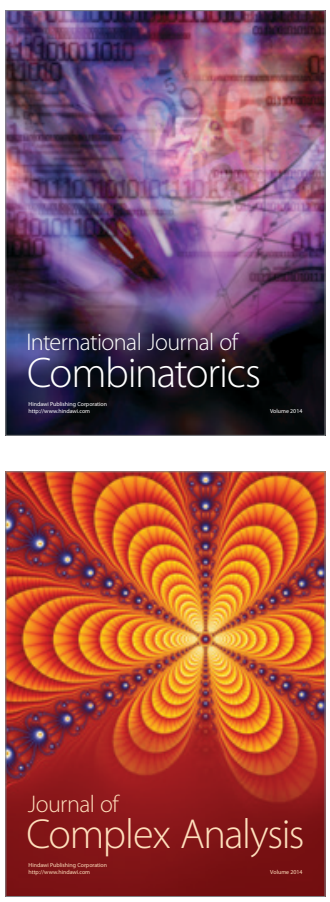

International Journal of

Mathematics and

Mathematical

Sciences
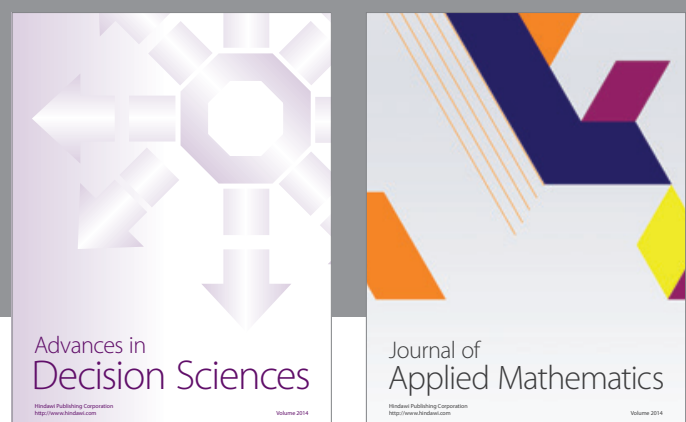

Journal of

Applied Mathematics
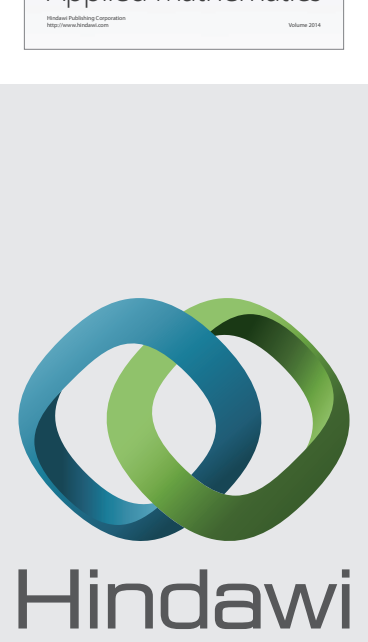

Submit your manuscripts at http://www.hindawi.com
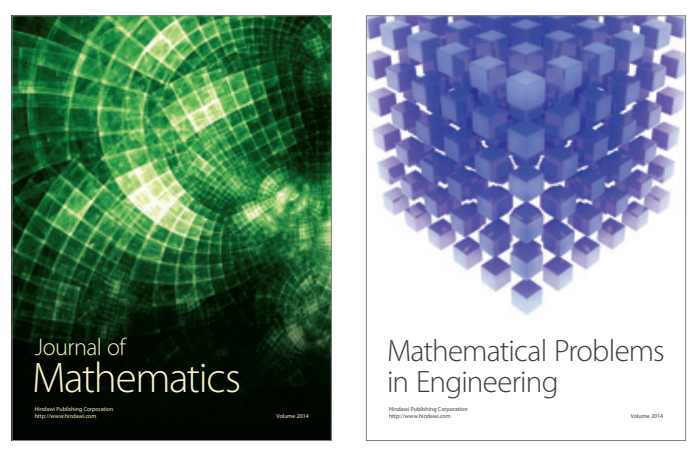

Mathematical Problems in Engineering
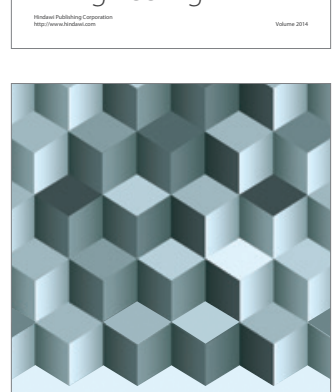

Journal of

Function Spaces
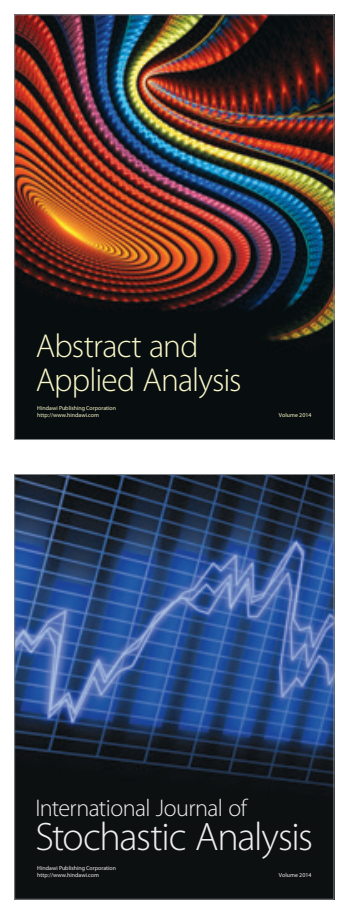

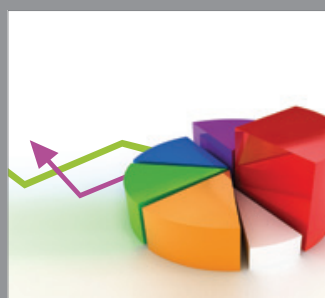

ournal of

Probability and Statistics

Promensencen
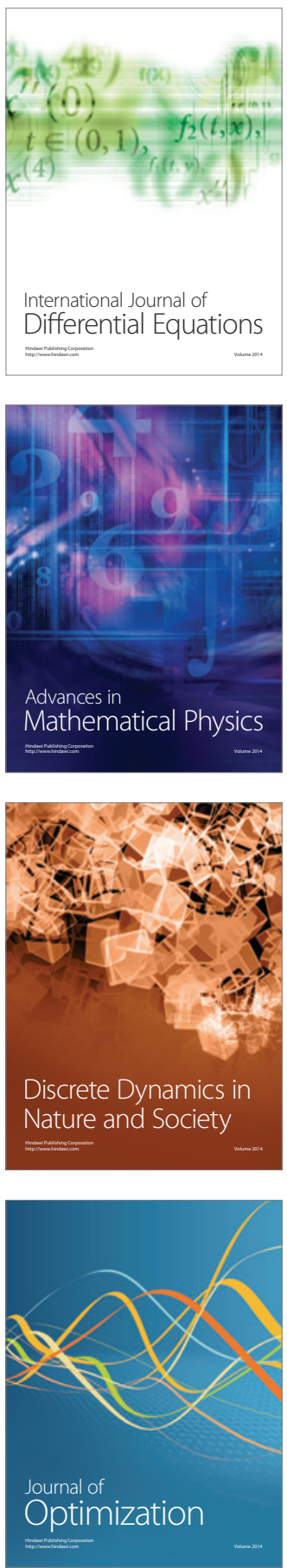